\title{
Stroke and myocardial infarction risk dependent on type of contraceptive pill
}

Increased risks of thrombotic stroke and myocardial infarction have been confirmed in women taking hormonal contraceptives by a group of researchers at the University of Copenhagen. Lindegaard et al. found that the risk of experiencing these vascular events was dependent on the dose of ethinylestradiol.

Other groups have assessed the effect of hormonal contraception on the risk of venous thromboembolism, but studies in which thrombotic stroke and myocardial infarction were the measured outcomes are scarce and their results are inconsistent. Although rarer, adverse events associated with arterial complications can be more serious than venous-associated events. Assessment of how oral contraceptive use influences a woman's risk of experiencing arterialassociated adverse events is therefore important to provide full information to women on their contraceptive options.

In this large-scale historical cohort study, records of $1,626,158$ women aged
15-49 years from a public registry were used. Patient records were excluded at the study outset if the individuals had prior gynaecologic, abdominal, breast, lung or haematologic cancer; venous or arterial thrombotic events; or cardiovascular disease. Additionally, the researchers censored from the analysis all data obtained during pregnancy and 3 months postpartum, during switches between contraceptive types, for 3 years following receipt of an intrauterine levonorgestrelreleasing device and while women underwent surgery or ovarian-stimulation therapy. Women who had undergone hysterectomy, bilateral oophorectomy or sterilization were excluded, and those who underwent these procedures during the study had their records censored following the interventions. In total, the records used amounted to 14,251,063 patient-years.

Oral contraceptives containing ethinylestradiol at $20 \mu \mathrm{g}$ or $30-40 \mu \mathrm{g}$ increased the risks of thrombotic stroke or myocardial infarction by $0.9-1.7$ or

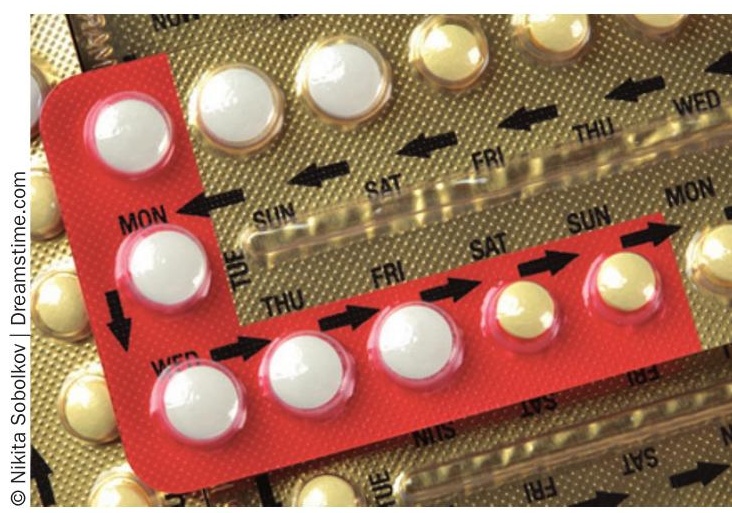

1.3-2.3 times, respectively. The researchers estimate that if 10,000 women use a contraceptive containing the progestin desogestrel and ethinylestradiol at a dose of $20 \mu \mathrm{g}$ for 1 year, two women will have arterial thrombosis.

Fiona Mitchell

Original article Lidegaard, $\emptyset$. et al. Thrombotic stroke and myocardial infarction with hormonal contraception. N. Engl. J. Med. 366, 2257-2266 (2012) 